\title{
BMJ Open Systematic scoping review protocol for clinical prediction rules (CPRs) in the management of patients with spinal cord injuries
}

\author{
Blake Boggenpoel, ${ }^{1}$ Vuyolwethu Madasa, ${ }^{1}$ Tarryn Jeftha, ${ }^{1}$ Conran Joseph ${ }^{1,2}$
}

To cite: Boggenpoel B, Madasa V, Jeftha T, et al. Systematic scoping review protocol for clinical prediction rules (CPRs) in the management of patients with spinal cord injuries. BMJ Open 2019;9:e025076. doi:10.1136/ bmjopen-2018-025076

- Prepublication history and additional material for this paper are available online. To view these files, please visit the journal online (http://dx.doi org/10.1136/bmjopen-2018025076).

Received 30 June 2018 Revised 1 October 2018 Accepted 7 December 2018

A) Check for updates

(C) Author(s) (or their employer(s)) 2019. Re-use permitted under CC BY-NC. No commercial re-use. See rights and permissions. Published by BMJ.

${ }^{1}$ Department of Physiotherapy, The University of the Western Cape, Cape Town, South Africa ${ }^{2}$ Department of Neurobiology, Care Sciences and Society, Physiotherapy Division, Karolinska Institutet, Stockholm, Sweden

Correspondence to Mr Blake Boggenpoel; bboggenpoel@uwc.ac.za

\section{ABSTRACT}

Introduction The upsurge in the use of clinical prediction models in general medical practice is a result of evidencebased practice. However, the total number of clinical prediction rules (CPRs) currently being used or undergoing impact analysis in the management of patients who have sustained spinal cord injuries (SCls) is unknown. This scoping review protocol will describe the current CPRs being used and highlight their possible strengths and weaknesses in SCI management.

Methods and analysis Arksey and 0'Malley's scoping review framework will be used. The following databases will be searched to identify relevant literature relating to the use of CPRs in the management of patients who have sustained an SCl: PubMed, Cumulative Index of Nursing and Allied Health Literature (CINAHL), ScienceDirect, EBSCOhost, Medline, OvidMedline and Google Scholar. Grey literature as well as reference lists of included studies will be searched. All studies relating to the use of CPRs in the management of patients with SCls will be included. Literature searches and data extraction will be performed independently by two groups of reviewers.

Ethics and dissemination Ethical clearance is not required for this scoping review study since only secondary data sources will be used. The findings of this review will be disseminated by means of peer-reviewed publication and conference proceedings. The final paper will be submitted for publication. Results of this review will also be presented at relevant conferences and disseminated to important stakeholders such as practicing physicians within specialised spinal care facilities within South Africa.

\section{INTRODUCTION}

The development of clinical prediction rules (CPRs) came about as a result of the drive for evidence-based practice. Novice clinicians often struggle with decision-making processes due to their lack of experience, while seasoned and more experienced clinicians neglect current evidence and base their knowledge on prior clinical situations. ${ }^{1}$ CPRs may be used to improve evidencebased decisions regarding patient care so as to improve patient prognosis. There are
Strengths and limitations of this study

- Clinical prediction rules (CPRs) are routinely used within the clinical setting; however, the actual number of validated prediction rules that exist for spinal cord injury (SCI) management is unknown.

- The review will also provide evidence of the psychometric properties of the CPRs, especially with regard to its clinical impact.

- A limitation of this review is that only English studies will be included, and this will affect our ability with regard to identifying relevant literature as certain studies might be overlooked.

- This will be the first review to map the current use of CPRs in the management of patients who have sustained an $\mathrm{SCl}$.

- The scoping review methodology will not allow one to assess the overall effectiveness of these CPRs in their respective settings.

three types of CPRs, which are namely diagnostic, prognostic and prescriptive/interventional in nature. Diagnostic rules, as the name suggests, help healthcare professionals diagnose a specific condition or complication. Prognostic rules provide guidance with regard to a patient's overall diagnosis or outcome, while prescriptive CPRs provide guidance with regard to the best treatment intervention to prescribe to a patient within a certain context. However, the development and use of prescriptive CPRs have noticeable flaws. ${ }^{2}$ A distinction has to be made between CPRs and decision-making tools as the manner in which impact is assessed differs. CPRs predict a relevant outcome, while the impact of clinical decision-making tools can only be assessed by the extent to which the CPR affects decision-making processes to improve patient outcomes. ${ }^{3}$ These CPRs may be used to 'manage patients' expectations and improve overall satisfaction; facilitate shared decision-making and counsel concerned patients on potential treatment 
options; identify strategies to optimise results; reduce heterogeneity of care and align surgeons' perceptions of outcome with objective evidence'. ${ }^{4}$ The use of these healthcare decision-making models or tools are also useful in ensuring quality improvement in the care that is being delivered; identifying environmental factors that need to be considered and the development of CPRs. ${ }^{56} \mathrm{~A}$ healthcare decision model is merely a way for healthcare practitioners to make an informed decision to address certain clinical questions that may need to be answered. It is further used to identify patients who are in need of scarce treatments and interventions, as well as for the allocation of resources.

The use of these prediction rules is important particularly decisions regarding high-risk patients, which are predominantly made subjectively. The latter is not ideal, since there are many factors that influence one's decision-making process such as values/norms and work experience. ${ }^{1}$ CPRs can be used as a means to help stratify patients with spinal cord injury (SCI) into either high or low risk, ${ }^{4}$ specifically in the case of patients that have suspected SCIs. For example, emergency care physicians and paramedics need to make rapid on the spot decisions whether to immobilise (ie, backboard, neck brace) a patient or not. However, immobilisation of these patients for a prolonged period of time may cause unnecessary discomfort, which should be avoided. ${ }^{7}$ CPRs are in most instances used in cases when confirming whether a patient has sustained a traumatic SCI or not following some sort of trauma. ${ }^{7}$ They are also used in determining the functional prognosis of these patients post injury. ${ }^{8-11}$ The use of decision-making tools to improve successful discharge from rehabilitation programmes has also been evaluated with positive outcomes. ${ }^{12}$ Although regaining function post injury is of importance for most patients, one will need to include outcome measures that are multidimensional in nature as patient priorities may differ. ${ }^{8}$ Besides preventing patient discomfort and improving decision-making within this specific context, the use of CPRs will help to reduce the financial burden on the healthcare system that is attached to the long-term care of these patients. ${ }^{37}$

The development of a CPR entails four distinct steps in its formulation.

Step 1: derivation; step 2: validation (narrow validation and broad validation); step 3: impact analysis and step 4: implementation. ${ }^{13-15}$ The final step of the clinical prediction model will entail the adoption of the model within the clinical setting. The final step might not be achievable immediately once the model has been developed and may take some time before it is incorporated into the clinical setting. However, if the impact of the tool shows positive results with regard to detecting high-risk patients, it will speed up the models uptake within the clinical setting. ${ }^{13-15}$ Very few CPRs actually reach this stage, which makes it difficult to determine whether a specific rule is effective within a specific clinical setting.
A preliminary search of the databases was done to identify any published or ongoing reviews regarding this topic, but none were found.

\section{Study rationale}

Due to the drive for evidence-based healthcare, one is in seeing an upsurge in the amount of CPRs which are being used to assist clinicians in their decision-making processes in general. Although this is the case, it is not always clear whether these CPRs have undergone sufficient psychometric testing to be considered valid and reliable. Currently, it is also unclear how many CPRs actually exist that may be used in the management of patients with SCI, and whether these rules can actually improve clinical decisions regarding the prognosis and diagnosis following SCIs. It will also allow us to identify any gaps in the literature regarding the use of CPR tools in the SCI population specifically looking at areas in which CPRs should be considered.

Hence, the research question of this review is to determine whether practical, validated CPRs exist for SCI in adult patients? The aim of this review is to describe the current use of CPRs in the management of patients with SCIs.

The following objectives have been set to achieve the desired aim of this review: (1) to document the settings in which the CPR is most commonly used within; (2) to document the end-users of the CPR (paramedic, trauma surgeon, physiotherapist, doctor, novice/or expert); (3) to describe the developmental process of the CPR (if documented); (4) to document whether the CPR is used for diagnostic, prognostic or prescriptive reasons; (5) to describe the impact of a CPR on the primary outcomes measured; (6) to describe the reliability, validity and impact of the CPR within each its given setting (ie, clinical sensibility) and (7) to describe how CPR reporting adheres to the Transparent Reporting of a multivariable prediction model for Individual Prognosis or Diagnosis (TRIPOD) statement. ${ }^{16}$

\section{METHODS}

The methodological framework that we will be following is according to the layout described in Arksey and O'Malley ${ }^{17}$ titled Scoping studies: towards a methodological framework. This method follows a five-stage process. Stage 1 involves identifying a research question; stage 2 involves identifying relevant studies; stage 3 involves the study selection; stage 4 involves charting of the data and stage 5 involves collating, summarising and reporting the results. This proposed review will make use of the standardised reporting guidelines for scoping reviews Preferred Reporting Items for Systematic Reviews and Meta-Analysis (PRISMA): extension for Scoping Reviews. ${ }^{18-20}$

\section{Search strategy}

The following databases will be searched from database inception till 2018 to identify relevant literature: 
PubMed, CINAHL, ScienceDirect, EBSCOhost, Medline, OvidMedline and Google Scholar. A preliminary search will be conducted to determine adequate search terms related to the review. Databases will be searched independently by two groups of reviewers (CJ and VM) and (BB and TJ). Duplicates will then be removed and titles and abstracts will be screened for their relevance. Once potentially relevant literature has been identified, the eligibility criteria listed below will be applied independently by each reviewer group. If a lack of consensus exists as to the inclusion of a specific paper, an external reviewer will then be consulted to make the final decision. In instances where consensus cannot be reached with all reviewers, then the article will be excluded from the study. References will be imported to Refworks, ${ }^{21}$ a reference managing software in specific folders based on database of retrieval. Relevant literature that has not been published will be identified by searching OpenGrey (www. opengrey.eu/), ${ }^{22}$ which is a database containing conference abstracts. Additional resources will be searched via ClinicalTrials.gov ${ }^{23}$ and WHO International Clinical Trials Registry Platform (ICTRP) ${ }^{24}$ in order to identify any ongoing and unpublished literature that could be of relevance to this review. All reference lists of articles that have been included in the review will also be searched independently by each reviewer group. A senior librarian at our home institution has been consulted to assist in our literature search and refine search terms. ${ }^{25}$

\section{Search terms (which include recognised medical subject heading} (MESH) terms and truncation)

"clinical prediction rule" OR "decision making model" OR "decision support techniques" OR "prediction" OR "predictive rule" AND "spinal cord injur*" AND non-traumatic spinal cord injur*

\section{Study selection}

The screening of the titles and abstracts and application of the eligibility criteria will be done independently by the two reviewer groups to minimise the chances of selection bias. Once titles and abstracts have been screened, the full-text versions of the potentially relevant literature will be retrieved. When the full-text articles of the relevant publications have been obtained, the inclusion and exclusion criteria as stated below will be applied. The study selection process will be documented making use of the PRISMA flow diagram. ${ }^{26}$

The inclusion criteria of the review are as follows: primary studies that include male and female participants ( $>18$ years of age) who have sustained a suspected traumatic (ie, high number of predictors which is indicative of an SCI) ${ }^{27}$ or non-traumatic SCI (ie, as result of some sort of pathology); studies published only in English (as no translation services will be available); studies published from database inception till 2018; studies incorporating all types of study designs (experimental and non-experimental); studies which focus on prognostic, diagnostic and prescriptive CPRs in the management of SCIs only; studies focusing on CPR use in acute, post-acute care, rehabilitation and follow-up care. The exclusion criteria will be limited to clinical commentaries. Eligibility could be changed during the course of the review as we get accustomed to the literature being reviewed. The latter is in line with standard scoping review methodology ${ }^{28}$ Included studies will be grouped based on whether or not they are describing a novel or validated CPR.

\section{Data extraction}

A data extraction form (see online supplementary material) from the Joanna Briggs Institute ${ }^{29}$ will be adapted and used to extract data from the included articles. This adapted data extraction sheet will be piloted independently by both reviewer groups (CJ and VM) and $(\mathrm{BB}$ and $\mathrm{TJ})$, to ensure that important results are captured consistently. After the piloting period has been completed, data extraction will be done independently by each reviewer group. If a lack of consensus exists with regard to the data that have been extracted, an external reviewer will be consulted to reach consensus. This form will be adapted to suit the requirements of this review and may undergo further changes during the course of the review as one becomes more accustomed to the content covered in the literature. ${ }^{28}$ The following data will be added to the Joanna Briggs Institute (JBI) data extraction sheet:

- Author(s), year of publication.

- Acute care or post-acute care (including rehabilitation and follow-up).

- End user (ie, profession using the CPR).

- Diagnostic, prognostic, prescriptive prediction rule (ie, type of prediction rule being used).

- CPR implementation process.

- Methodology (ie, type of study design).

- Outcome measures used (ie, primary outcome measures being assessed).

- Effect of the CPR on primary patient outcomes being assessed.

- Psychometric validation of the tool (ie, validity and reliability).

- Clinical sensibility.

- Study limitations.

The final stage of the scoping review process will involve summarising, collating and reporting results as laid out in Arksey and O'Malley. The results of this systematic scoping review will be aggregated to give the end user a better idea of what clinical prediction tools are available to best manage persons who have sustained an SCI, and whether these rules have gone through the necessary developmental processes to be considered valid and reliable. The latter will also allow us to identify possible gaps with regard to the areas in which CPRs should be developed. The results will be conveyed in a tabular and narrative format, and be presented under the four main headings mentioned below.

- Description of the CPR used. 
- Developmental process of the CPR (which will include possible methodological strengths and flaws).

- Psychometric properties relating to the CPR.

- Impact of the CPR on the primary outcome measures being assessed.

- Consistency of CPR reporting with respect to the TRIPOD statement.

\section{PATIENT AND PUBLIC INVOLVEMENT}

Due to the evidence-based approach currently being used in general medicine, and the high costs involved in managing patients that have sustained SCIs, CPRs can assist in reducing the costs placed on healthcare facilities, while at the same time improving patient prognosis. Patients were not involved in the study design besides the initial conceptualisation of the research question.

\section{CONCLUSIONS}

This systematic scoping review will allow us to map the current use of CPRs and their impact on clinically important outcome measures specifically relating to the SCI population. This is particularly important due to the fact that SCIs are not really declining, injured individuals are continuing to die and develop secondary conditions following injury, and healthcare resources are continuously being cut which necessitates practitioners to develop and implement cost-effective care packages for this vulnerable group.

Contributors BB conceived the idea and decided upon the research question and objectives that were outlined along with drafting and editing the final protocol. CJ assisted in refining objectives for the review and assisted with editing and drafting of the final protocol. VM and TJ assisted with editing of the final protocol. All authors will be responsible for conducting the literature searches, data extraction and write up of the final research paper.

Funding This proposed scoping review forms part of a larger project, aimed at strengthening health systems for people with spinal cord injury, which received a 5 -year grant from the South African Medical Research Council within the Research Capacity Development Initiative.

Competing interests None declared.

Patient consent for publication Not required.

Provenance and peer review Not commissioned; externally peer reviewed.

Open access This is an open access article distributed in accordance with the Creative Commons Attribution Non Commercial (CC BY-NC 4.0) license, which permits others to distribute, remix, adapt, build upon this work non-commercially, and license their derivative works on different terms, provided the original work is properly cited, appropriate credit is given, any changes made indicated, and the use is non-commercial. See: http://creativecommons.org/licenses/by-nc/4.0/.

\section{REFERENCES}

1. Smith M, Higgs J, Ellis E. Factors influencing clinical decision making, Clinical reasoning in the health professions. 2008;3:89-100.

2. Cook CE. Potential pitfalls of clinical prediction rules. J Man Manip Ther 2008;16:69-71.

3. Reilly BM, Evans AT. Translating clinical research into clinical practice: impact of using prediction rules to make decisions. Ann Intern Med 2006;144:201-9.
4. Tetreault L, Le D, Côté P, et al. The practical application of clinical prediction rules: A commentary using case examples in surgical patients with degenerative cervical myelopathy. Global Spine $J$ 2015;5:457-65.

5. Bae JM. The clinical decision analysis using decision tree. Epidemiol Health 2014;36:e2014025.

6. McGinn TG, Guyatt GH, Wyer PC, et al. Users' guides to the medical literature: XXII: how to use articles about clinical decision rules. JAMA 2000;284:79-84.

7. Vaillancourt C, Charette M, Kasaboski A, et al. Evaluation of the safety of C-spine clearance by paramedics: design and methodology. BMC Emerg Med 2011;11:11:1.

8. van Middendorp JJ, Hosman AJ, Donders AR, et al. A clinical prediction rule for ambulation outcomes after traumatic spinal cord injury: a longitudinal cohort study. Lancet 2011;377:1004-10.

9. Hicks KE, Zhao Y, Fallah N, et al. A simplified clinical prediction rule for prognosticating independent walking after spinal cord injury: a prospective study from a Canadian multicenter spinal cord injury registry. Spine J 2017;17:1383-92.

10. van Silfhout L, Peters AE, Graco M, et al. Validation of the Dutch clinical prediction rule for ambulation outcomes in an inpatient setting following traumatic spinal cord injury. Spinal Cord 2016;54:614-8.

11. Wilson JR, Grossman RG, Frankowski RF, et al. A clinical prediction model for long-term functional outcome after traumatic spinal cord injury based on acute clinical and imaging factors. $J$ Neurotrauma 2012;29:2263-71.

12. Burns AS, Yee J, Flett HM, et al. Impact of benchmarking and clinical decision making tools on rehabilitation length of stay following spinal cord injury. Spinal Cord 2013;51:165-9.

13. Adams ST, Leveson SH. Clinical prediction rules. BMJ 2012;344:d8312.

14. Laupacis A, Sekar N, Stiell IG. Clinical prediction rules. A review and suggested modifications of methodological standards. JAMA 1997;277:488-94.

15. Toll DB, Janssen KJ, Vergouwe Y, et al. Validation, updating and impact of clinical prediction rules: a review. J Clin Epidemiol 2008;61:1085-94.

16. Collins GS, Reitsma JB, Altman DG, et al. Transparent reporting of a multivariable prediction model for individual prognosis or diagnosis (TRIPOD): the TRIPOD statement. BMC Med 2015;13:1.

17. Arksey H, O'Malley L. Scoping studies: towards a methodological framework. Int J Soc Res Methodol 2005;8:19-32.

18. Tricco A, Straus S, Moher D. Preferred reporting items for systematic reviews and meta-analysis: extension for Scoping Reviews (PRISMAScR), EQUATOR Netw. 2017. http://www.equator-network.org/ wp-content/uploads/2009/02/Executive-summary_ScR_Dec-9.pdf (accessed 27 June 2018).

19. Tricco AC, Lillie E, Zarin W, et al. A scoping review on the conduct and reporting of scoping reviews. BMC Med Res Methodol 2016;16:15.

20. Tricco AC, Lillie E, Zarin W, et al. PRISMA Extension for Scoping Reviews (PRISMA-ScR): Checklist and Explanation. Ann Intern Med 2018;169:467.

21. RefWorks-COS PL, ProQuest L. RefWorks, Ann Arbour: ProQuest LLC, 2001.

22. OpenGrey. System for information on grey literature in europe. 2018 http://www.opengrey.eu/

23. U.S National Library of Medicine. ClinicalTrials.gov. https:// clinicaltrials.gov/ct2/home/

24. WHO. International Clinical Trails Registry Platform. 2018 https:// www.who.int/ictrp/en/

25. George G, Ferguson LA, Pearce P. Finding a needle in the haystack: performing an in-depth literature search to answer a clinical question. Nursing 2014;4:65-76.

26. Moher D, Liberati A, Tetzlaff J, et al. Preferred reporting items for systematic reviews and meta-analyses: the PRISMA statement. Int $J$ Surg 2010;8:336-41.

27. Oteir AO, Smith K, Stoelwinder J, et al. Prehospital predictors of traumatic spinal cord injury in Victoria, Australia. Prehosp Emerg Care 2017;21:583-90.

28. Levac D, Colquhoun H, O'Brien KK. Scoping studies: advancing the methodology. Implement Sci 2010;5:69.

29. Peters M, Godfrey C, Mclnerney P, et al. Chapter 11: Scoping Reviews, Joanna Briggs Institute Reviewer Manual [Internet]. Adelaide (AU): The Joanna Briggs Institute, 2017. 